\title{
Les productions céramiques du Chasséen méridional entre l'Hérault et l'Orb : nouvelles séquences typochronologiques
}

\section{Muriel Gandelin et Hélène Vergély}

\section{(2) OpenEdition}

Journals

Édition électronique

URL : https://journals.openedition.org/archeopages/475

DOI : 10.4000/archeopages. 475

ISSN : 2269-9872

Éditeur

INRAP - Institut national de recherches archéologiques préventives

Édition imprimée

Date de publication : 1 janvier 2012

Pagination : 88-95

ISSN : 1622-8545

\section{Référence électronique}

Muriel Gandelin et Hélène Vergély, «Les productions céramiques du Chasséen méridional entre I'Hérault et l'Orb : nouvelles séquences typochronologiques », Archéopages [En ligne], Hors-série 3 | 2012, mis en ligne le 01 janvier 2012, consulté le 26 février 2023. URL : http://

journals.openedition.org/archeopages/475; DOI : https://doi.org/10.4000/archeopages.475 

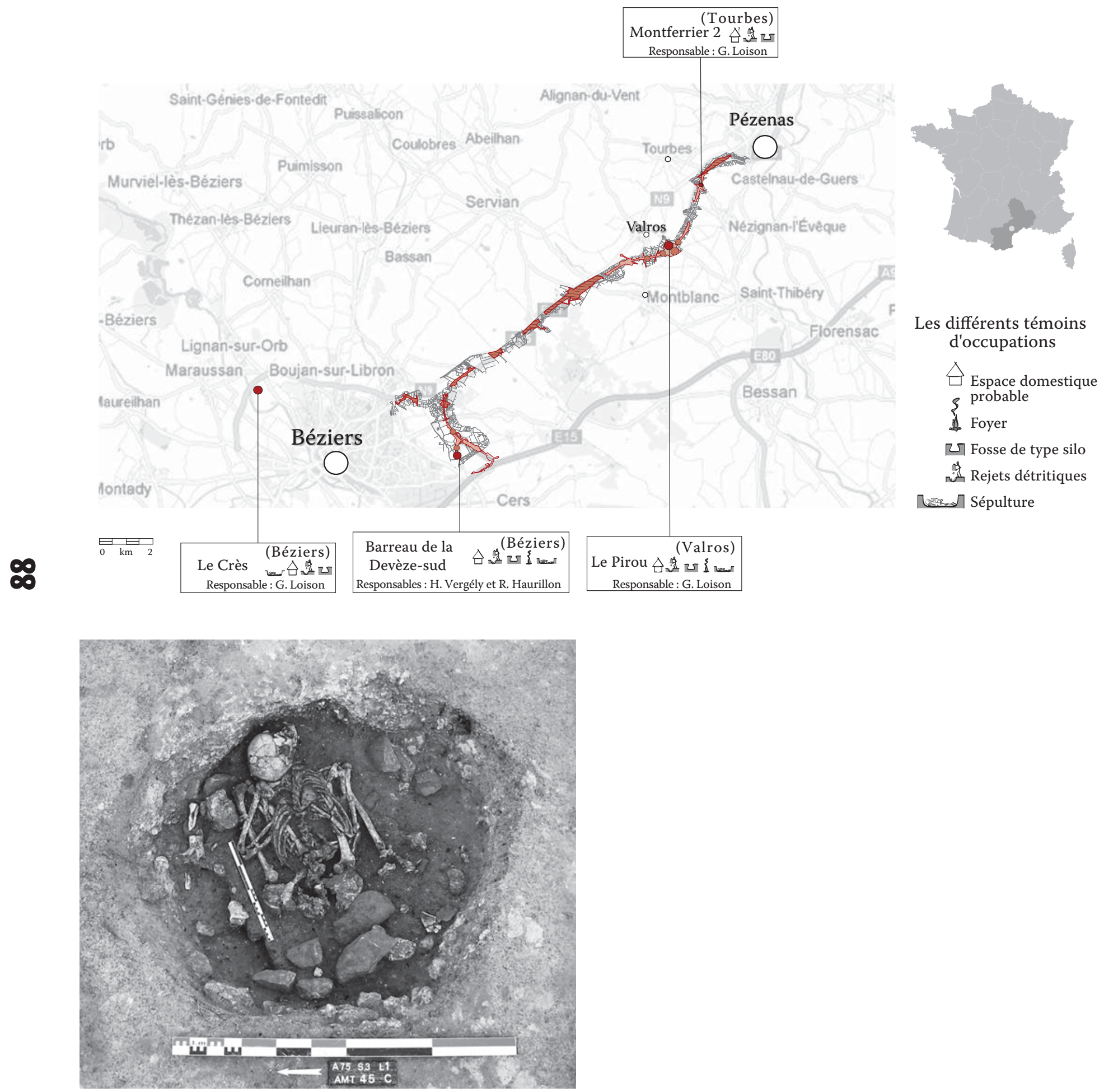

[Fig.1] Carte de localisation des principaux sites chasséens fouillés dans l'Ouest de l'Hérault au cours de la dernière décennie.
[Fig.2] Sépulture du Chasséen ancien du Pirou (Béziers). 
très limitées : une vingtaine de sépultures pour plus de deux mille structures archéologiques fouillées. La coexistence habitat/sépulture est toutefois récurrente dans tout le Midi de la France en contexte chasséen sans qu'il soit réellement possible de déterminer s'il s'agit d'une pratique opportuniste ou d'une pratique codifiée réservée à certains défunts. Il apparaît toutefois clairement, dans le Toulousain, que les sépultures à «creusement spécifiquement funéraire» du type de celle de Sauzas sont beaucoup plus richement dotées que les sépultures installées dans des structures en réemploi; bien que ces dernières ne soient pas exemptes d'offrandes. Alors que le premier type concentre l'essentiel du mobilier (8 tombes regroupant 55 objets, soit une moyenne de 7 objets par sépulture), le second (23 structures funéraires et 43 objets, soit une moyenne de 2 objets par sépulture) est beaucoup plus pauvre et la céramique, seul élément attestant clairement d'un éventuel viatique, $y$ demeure très indigente. Aussi peut-on s'interroger sur la différence de statut qui pouvait exister entre les individus inhumés dans les fosses réemployées, pauvres en mobilier, et ceux inhumés dans les aménagements spécifiquement sépulcraux au sein de zones réservées, avec un mobilier funéraire beaucoup plus abondant. La possibilité de la présence d'un enfant subordonné à un adulte masculin dans l'une des sépultures de Sauzas est également un fait nouveau qui relance le débat sur l'existence possible de morts «d'accompagnement » en contexte chasséen garonnais.

La mise au jour de la zone sépulcrale de Sauzas s'inscrit dans une dynamique plus générale liée aux nombreux travaux récents d'aménagement du territoire toulousain qui ont conduit, ces dernières années, à plusieurs découvertes majeures réalisées par les équipes de l'Inrap. Pour la période du Néolithique moyen, la fouille récente de l'enceinte de Château-Percin (Gandelin et al., sous presse) et la mise en évidence de son caractère comminatoire, voire défensif, ont permis d'envisager l'existence d'une société potentiellement instable, peut-être soumise à des conflits. Dans ce contexte, la distinction de certains individus et la mise en place progressive d'une hiérarchie sociale peuvent être envisagées. L'apparition de biens de prestige mais aussi la différence de traitement post mortem, telle qu'elle a été mise en évidence grâce aux fouilles de Sauzas, pourraient ainsi témoigner de ce phénomène. La multitude et la complexité des facteurs qui sous-tendent les pratiques funéraires ne permettent toutefois pas, à l'heure actuelle, de mettre en regard la variété des modes sépulcraux et l'organisation sociale des groupes néolithiques méridionaux.

Cap-Jedikian G., Perrin T., Remicourt M., Servelle C., 2008 : «Révision des données disponibles sur les aménagements funéraires du site de Saint-Michel-du-Touch (Toulouse, Haute-Garonne) », in Vaquer J., Gandelin M., Remicourt M., TCheremissinoff Y. (Dir.), p. $180-196$.

Gandelin M., Pons F., De Chazelles C.-A., Sous presse : « L'enceinte chasséenne de Château-Percin. Un témoignage exceptionnel d'architecture monumentale néolithique en Haute-Garonne », Archéopages, 33, p. 12-17.

VAquer J., Gandelin M., Remicourt M., TCheremissinoff Y.( Dir.), 2008 : Défunts néolithiques en Toulousain. Toulouse, Archives d'écologie Préhistoriques, 234 p.
Les productions céramiques du Chasséen méridional entre l'Hérault et l'Orb : nouvelles séquences typochronologiques

Muriel Gandelin, Hélène Vergély

Inrap, UMR 5608 "Travaux et Recherches Archéologiques sur les Cultures, les Espaces et les Sociétés»

C es dix dernières années, les connaissances relatives au Chasséen de la partie occidentale de l'Hérault ont considérablement évolué grâce à plusieurs fouilles archéologiques majeures opérées par des équipes de l'Inrap. À l'échelle régionale, les découvertes réalisées dans le cadre de ces fouilles préventives prennent toute leur importance, puisqu'elles viennent combler un déficit de documentation archéologique relatif à l'évolution typochronologique des productions céramiques du Chasséen méridional dans les bassins de l'Orb et de l'Hérault [Fig.1].

2001-2011: 10 ans de découvertes. La fouille du site du Crès (Béziers), achevée en 2001, avait marqué un tournant dans la recherche sur le Chasséen méridional, ce site ayant livré, à lui seul, davantage de sépultures du début du Néolithique moyen que l'ensemble des occupations connues jusque-là dans tout le Sud de la France. La présence d'un espace funéraire à part entière, situé à proximité directe d'un habitat, représentait un fait nouveau et d'importance pour les préhistoriens. Le site du Crès a également livré d'abondants vestiges mobiliers qui constituaient, au moment de leur découverte, un premier jalon pour la connaissance des productions domestiques du Chasséen ancien de la zone biterroise jusque-là fort mal connue.

Quelques années plus tard, à la faveur des travaux d'aménagement de l'Autoroute A75 entre Béziers et Pézenas, plusieurs découvertes conséquentes sont venues compléter cette séquence chronologique. Le site du Pirou à Valros, fouillé entre octobre 2006 et juillet $2007^{2}$, a livré les vestiges d'une occupation du Chasséen ancien (4500-410o av. notre ère) qui témoignent de la présence d'un habitat intégrant le fait funéraire, bien que dans une proportion moindre qu'au Crès. Sur les 160 structures chasséennes reconnues, sept contenaient une sépulture et une dizaine ont livré le dépôt d'un chien inhumé selon des modalités très proches de celles observées pour les êtres humains [Fig.2]. Le site a également livré un mobilier abondant et très homogène qui permet, notamment, de caractériser les plus anciennes productions céramiques chasséennes connues dans ce secteur. La série céramique du Pirou est, quantitativement, la plus importante actuellement connue pour le Chasséen ancien de cette zone géographique. À quelques kilomètres à peine, sur la commune de Tourbes, une petite occupation (Montferrier 2) a livré une vingtaine de fosses également rattachées au Chasséen ancien ${ }^{3}$. La petite série céramique découverte sur ce site présente de nombreuses affinités avec celle du Crès et permet, mise en regard 


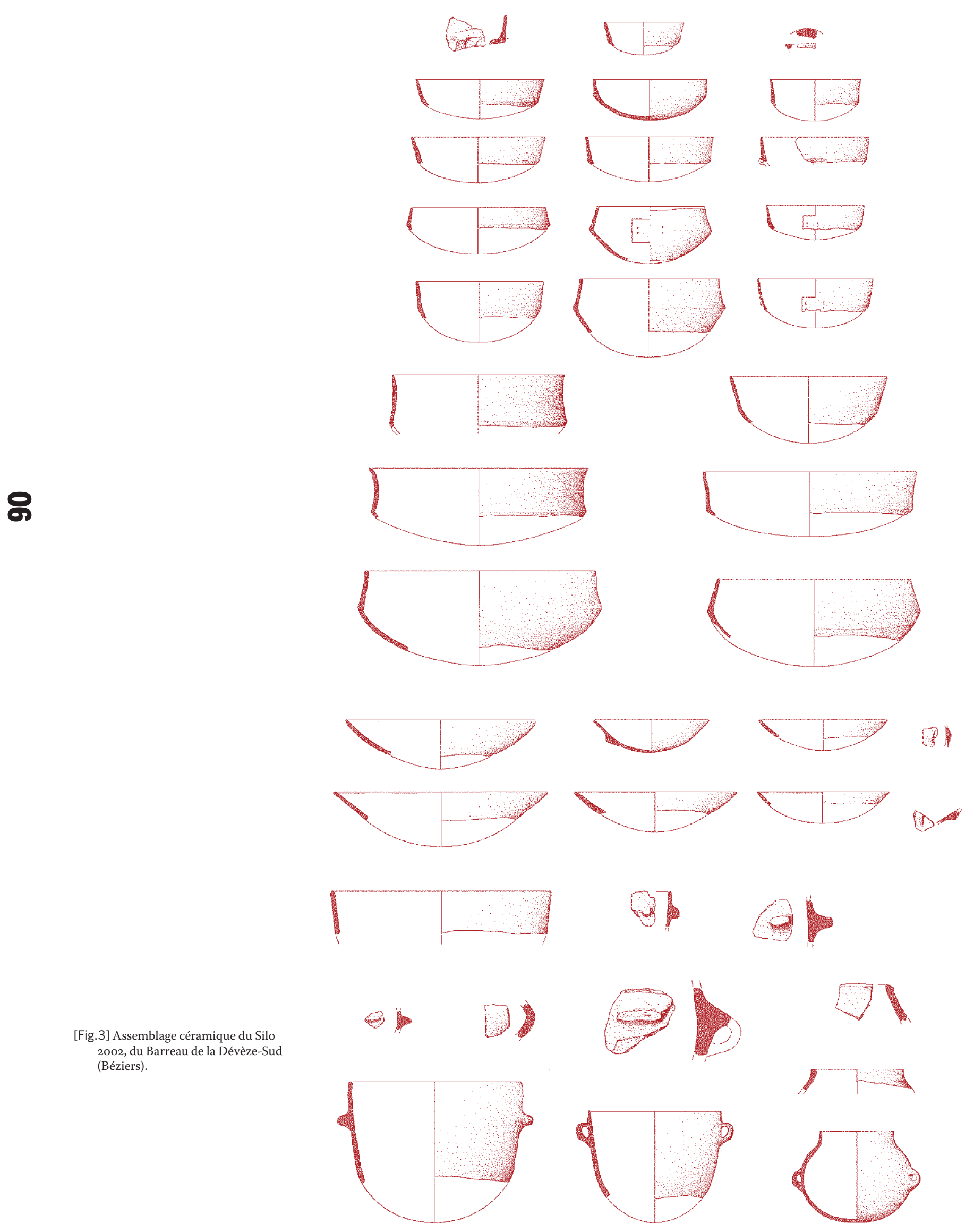


de la série du Pirou, de percevoir une évolution au sein des productions du Chasséen ancien de la zone piscénoise.

En 2008, les dernières fouilles réalisées dans le cadre de l'A75, au Barreau de la Devèze-Sud à Béziers $\mathbf{4}$, sont à l'origine de la découverte de plusieurs occupations chasséennes, dont les principales se rapportent aux phases classique et récente de cette culture, documentées pour la première fois de façon conséquente à l'ouest de l'Hérault. Ce site est remarquable, notamment par l'abondance des vestiges récoltés au sein d'un nombre limité d'aménagements domestiques qui témoignent de plusieurs occupations successives [Fig.3]. Il a également livré une sépulture tout à fait exceptionnelle pour la période chasséenne [cf. encadré p 95]. À l'issue de ces découvertes, il est possible de proposer une première synthèse de l'évolution typochronologique des productions céramiques du Chasséen méridional héraultais, au sein d'une zone située entre les fleuves Hérault et Orb [Fig.4].

Le Chasséen ancien. La série de mobilier céramique issue des fouilles du Pirou constitue un ensemble quantitativement important : 12500 tessons, soit $120 \mathrm{~kg}$ de céramique, qui ont permis d'individualiser 180 vases et plus d'un millier de fragments isolés présentant une spécificité typologique (bords, éléments de préhension, décors isolés). La série du Crès représente plus de 6 ooo tessons, soit plus de $80 \mathrm{~kg}$ de mobilier, parmi lesquels 80 formes individualisables et 694 fragments typiques ont été étudiés par G. Jédikian (2004).

Considérées dans leur ensemble, les productions céramiques du début du Chasséen présentent presque exclusivement des profils continus. Les formes segmentées sont absentes ou très rares, à l'exception des assiettes à marli bien différencié et des coupes à socle. Les coupes en calotte sont bien attestées, elles représentent plus du tiers des formes individualisables au Pirou. Elles sont exclusivement sans sillon, parfois ornées d'un bouton. De rares exemplaires (un est attesté au Crès, un autre au Pirou) sont dotés d'anses en ruban diamétralement opposées. Les formes globuleuses profondes dominent largement le corpus des deux sites. Les vases les plus petits présentent fréquemment un profil galbé. Les modes de suspension associés sont, le plus souvent, constitués de barrettes ou de cordons multiforés placés dans la partie supérieure des vases. Il semble que la localisation de ces cordons directement sur la lèvre, fréquente au Crès et à Montferrier 2, et absente sur le site du Pirou, certainement plus ancien, constitue une évolution du type. Sur les vases de moyen et grand volume, la quasi-totalité des préhensions est assurée par des anses en ruban, parfois funiculaires et, plus rarement, par des anses en boudin. Les languettes et tétons de préhension sont totalement absents. Des bandeaux ou des épaississements viennent parfois renforcer les bords extérieurs des vases globuleux.Des plaquettes au faible relief, larges et perforées horizontalement, ornent parfois le maximum des panses des vases à fond surbaissé et parois convergentes, dont la morphologie et la taille annoncent les formes carénées qui apparaîtront plus tard. On retrouve aussi ce type d'applique sur le bas des panses de formes à parois divergentes au Crès.

La famille des vases à col occupe toujours moins de 5\% des assemblages. Elle n'est documentée que par des exemplaires à col court et mal différencié de la panse. Les coupes à socle sont circulaires, d'un diamètre généralement compris entre 10 et $15 \mathrm{~cm}$ et d'une hauteur toujours supérieure à la moitié du diamètre au bord. Leur profil est droit, ou plus ou moins concave. Elles sont fréquemment décorées sur le pied et sur le marli par des motifs incisés organisés en réseaux géométriques plus ou moins réguliers. Les louches à poignée plate, parfois relevée, sont fréquentes. Les décors, rares, se concentrent sur les marlis des assiettes et les parois des coupes à socle. Il s'agit essentiellement de motifs en sillons ou en micro-sillons figurant des triangles hachurés, des chevrons et plus rarement des damiers intégrés à des cloisonnements triangulaires, un motif en échelle et de possibles fragments de décors cannelés « en guirlande» sont également attestés au Pirou [Fig.5]. Au Crès, un vase à carène très haute et anse tunelliforme verticale témoigne clairement d'une influence ou d'une relation avec le groupe culturel Montbolo, toutefois cette affinité est absente au Pirou.

La légère diachronie qui existe entre les séries du Crès, datée entre 4350 à 4.000 av. notre ère, et du Pirou, datée entre 4.450 et 4050 (mais probablement antérieure à 4,200 av. notre ère), permet d'ores et déjà de percevoir une évolution au sein des productions du Chasséen ancien avec notamment une première phase qui se distinguerait de la suivante par l'absence totale des carènes anguleuses et des cordons multiforés disposés directement sur la lèvre des vases, ainsi que par un galbe plus systématique des profils. Toutefois, les similitudes entre les deux séries restent très nombreuses et témoignent d'une évolution relativement lente des formes et des décors entre 4500 et 4.100 av. notre ère.

Les convergences entre les séries du Chasséen ancien héraultais et du faciès audois, principalement documenté par le style des Plots à Berriac, sont très fortes, bien que des affinités existent également avec les productions héraultaises plus orientales (Vaquer, 1991 ; Georjon, 2003).

Le Chasséen «classique». La phase médiane du Chasséen dite «classique», bien définie dans l'Aude et le Toulousain, n'est pas toujours individualisée au sein des productions plus orientales, pour lesquelles une bipartition ancienne/récente est le plus souvent proposée. Pourtant, le corpus du Barreau de la Devèze-Sud, qui associe des séries biens documentées issues d'ensembles clos et des dates ${ }^{14} \mathrm{C}$ fiables, autorise clairement la distinction de deux phases successives, postérieures au Chasséen ancien, sur le modèle actuellement en vigueur dans les Chasséens garonnais et audois. Par définition, la phase médiane du Chasséen comporte de nombreux éléments communs, d'une part, à la phase ancienne et, d'autre part, à la phase récente.

Traditionnellement distinguée de la phase 


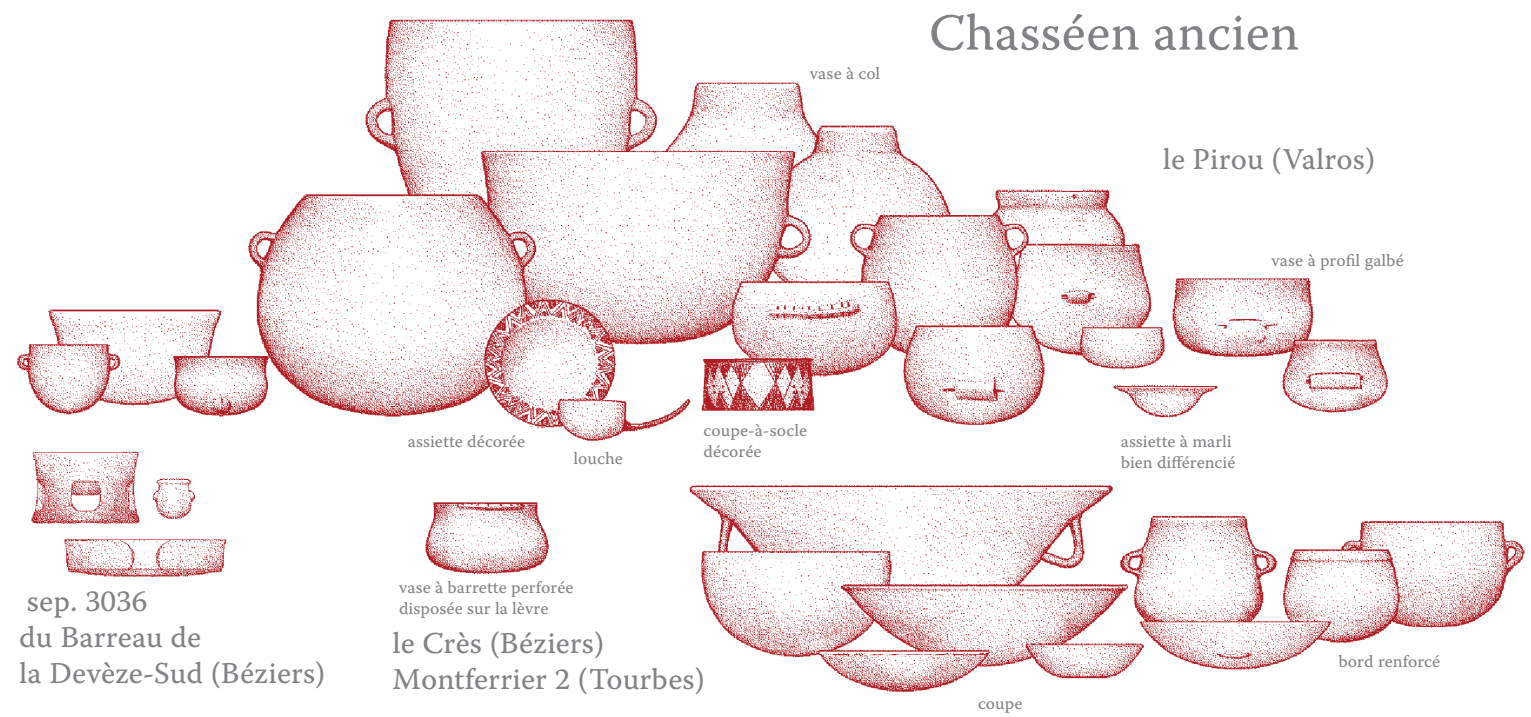

\section{Chasséen classique}

ชิ

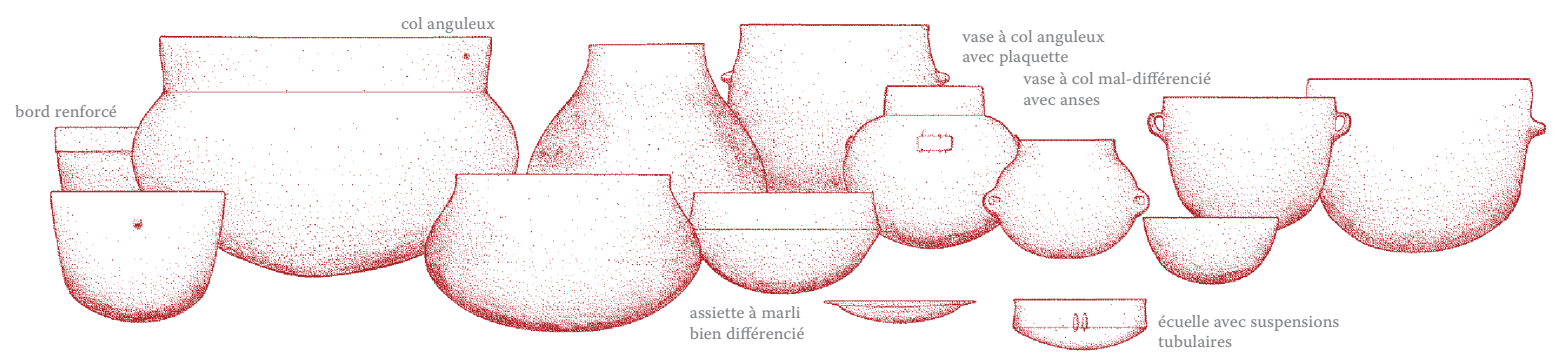

Barreau de

la Devèze-Sud (Béziers)
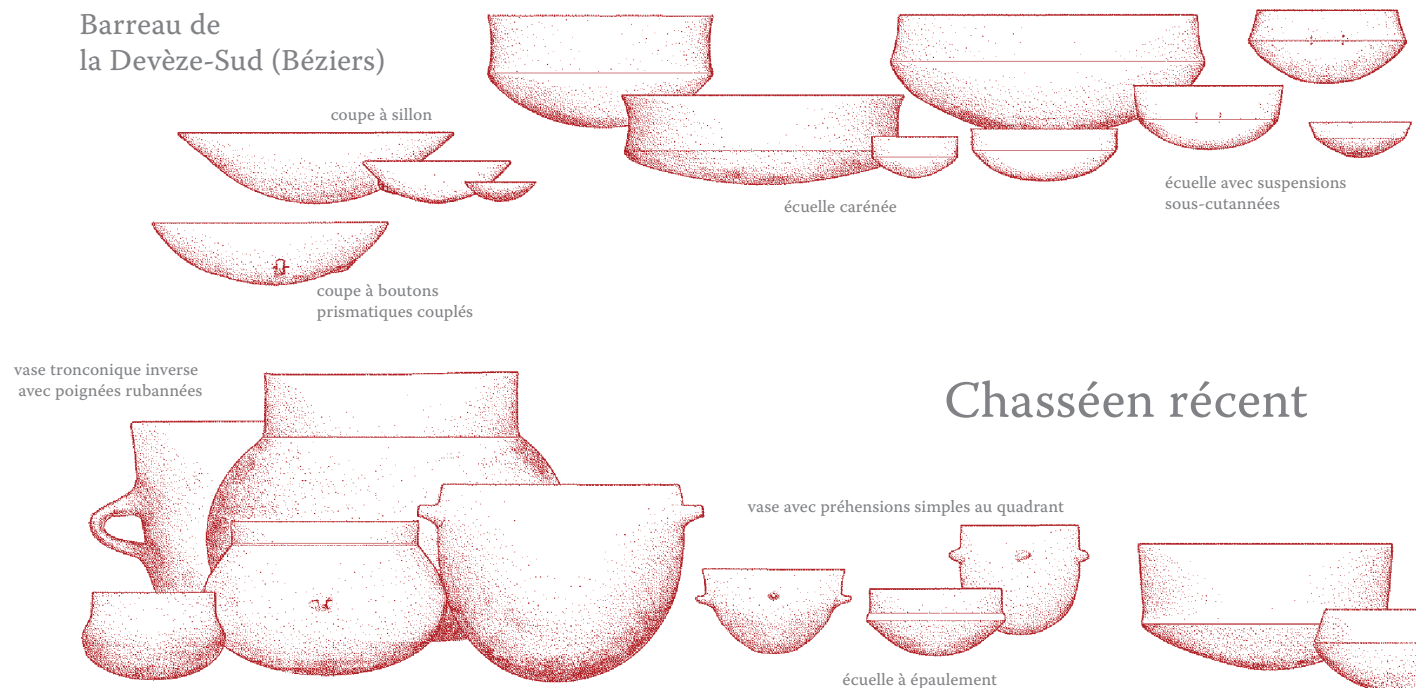

\section{Chasséen récent}
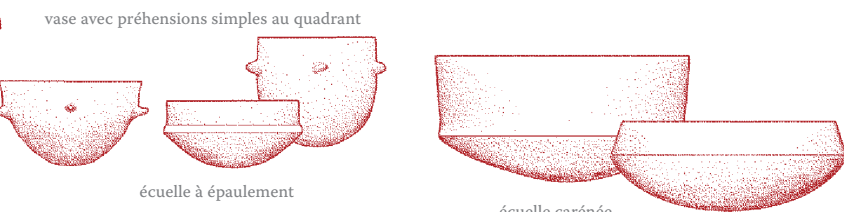

[Fig.4] Vue synoptique de l'évolution des productions céramiques chasséennes entre Hérault et Orb.

écuelle avec bouton prismatique
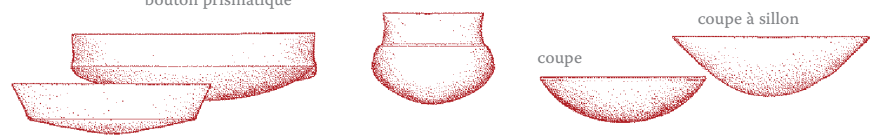
ancienne par la présence de coupes à sillon périphérique interne, le Chasséen classique en conserve toutefois la plupart des morphotypes : anses en ruban, formes galbées, éléments horizontaux perforés verticalement... Cependant, la proportion de ces éléments, concurrencés par de nouvelles formes, tend fortement à diminuer. Certains types voient également leur proportion augmenter de façon très significative au sein des assemblages. Ainsi, les formes segmentées, notamment les carènes vives, très rares dans la phase précédente, y sont très bien représentées au détriment des vases à profil galbé et à carène douce dont la proportion devient anecdotique. Les languettes et mamelons de préhension font leur apparition sur les vases de moyens et gros volumes, et concurrencent fortement les anses en ruban qui restent toutefois fréquentes. Les vases à col, nettement plus présents que durant le Chasséen ancien, adoptent plus souvent des raccords colpanse anguleux. Traditionnellement, dans l'Aude et le Toulousain notamment, les décors sont bien plus fréquents durant cette phase que durant les deux autres. Cette période «classique» est calée chronologiquement à la charnière des $\mathrm{V}^{\mathrm{e}}$ et $\mathrm{IV}^{\mathrm{e}}$ millénaires, entre environ 4100 et $3850 \mathrm{av}$. notre ère.

$\mathrm{Au}$ Barreau de la Devèze-Sud, huit fosses, dont quatre, riches en mobilier, qui ont fait l'objet d'une datation radiocarbone, s'y rattachent. Les datations ${ }^{14} \mathrm{C}$ réalisées permettent de caler cette période d'occupation du site entre 4075 et 3950 av. notre ère. Au stade actuel des connaissances, cette série constitue le corpus le mieux documenté relatif au Chasséen classique dans la zone biterroise. Sa qualité réside notamment dans la présence de deux assemblages, homogènes et très conséquents, issus de deux fosses qui ont livré, chacune, plus d'un millier de tessons dont une soixantaine d'éléments typiques, parmi lesquels un nombre important de vases restituables. La plupart des formes spécifiques du Chasséen ancien sont ici quasiment absentes, toutefois, quelques éléments comme les anses en ruban perdurent. Ces dernières sont néanmoins concurrencées par les préhensions simples, tétons et languettes, parfois perforées et généralement disposées sur le haut des vases de moyen et grand volume. Les carènes anguleuses sont nombreuses et les vases à col segmenté sont présents bien qu'en faible proportion. Telle qu'elle apparait au Barreau de la Devèze-Sud, la phase médiane du Chasséen de la basse vallée de l'Hérault présente de nombreux critères communs avec les séries «classiques» de l'Aude et du Toulousain. Si l'on considère les quatre grandes familles de vases, on observe un assemblage largement dominé par les écuelles carénées,

les coupes venant en seconde position, suivies par les vases à col et les globuleux en quantité voisine. Évidemment, cette répartition est partiellement induite par la fragmentation, qui favorise les formes segmentées (écuelles carénées, cols anguleux) qui se multiplient à l'approche du $\mathrm{IV}^{\mathrm{e}}$ millénaire. Toutefois, si l'on met ces données en parallèle avec celles issues de plusieurs grands ensembles chasséens méridionaux bien documentés et bien datés, il apparait que la série du Barreau de la
Devèze-Sud s'inscrit parfaitement dans le schéma observé par ailleurs [Fig.6]. Cette projection rapide, nonobstant des données relativement disparates provenant de sites parfois éloignés, montre bien que, de façon globale, la série du Barreau de la Devèze-Sud présente les mêmes caractéristiques générales que les autres ensembles du Chasséen classique de l'Aude et du Toulousain. Des parallèles nombreux existent également avec les séries plus orientales appartenant au même horizon chronologique. On note toutefois, comme plutôt caractéristique de la série biterroise, la rareté des décors et la monotonie des systèmes de suspension ou encore la taille relativement importante de certains vases à col et de certaines écuelles carénées dont le diamètre au bord peut dépasser les $40 \mathrm{~cm}$.

Le Chasséen « récent ». Le Chasséen récent était jusquà présent inconnu ou très mal défini dans la zone biterroise. La série du Barreau de la DevèzeSud, rattachée à cette période malgré un effectif relativement faible, constitue donc un apport conséquent pour la définition et la connaissance de l'évolution régionale du Chasséen de la basse vallée de l'Hérault. Dans le Toulousain et dans l'Aude, où cette phase est bien définie, le Chasséen récent se caractérise principalement par la proportion importante de formes segmentées (carènes anguleuses, cols bien différenciés des panses), la variété des types de raccords cols/panses avec les «pseudo-cols» et les «micro-épaulements » bien représentés, la présence d'écuelles carénées à épaulement, parfois dotées d'une unique anse rubanée, la fréquence de préhensions simples, la rareté des décors ainsi que celle des anses en ruban.

Au Barreau de la Devèze-Sud, une fosse datée entre 3932 et 3538 av. notre ère, avec un maximum de probabilité entre 3800 et $3633^{5}$, se rattache clairement à cette occupation et au moins deux autres aménagements présentent un ou plusieurs éléments qui autorisent un rattachement au Chasséen récent. Au sein de cette petite série, on retrouve notamment, parmi les critères retenus comme caractéristiques du Chasséen récent dans l'Aude et le Toulousain : l'existence d'écuelle à épaulement; la proportion importante des formes segmentées, et notamment de carènes anguleuses et de cols bien différenciés des panses ; la variété importante des types de raccords cols/panses sur les vases à col avec les « micro-corniches » bien représentéés; la fréquence des languettes et tétons et la rareté des anses. Comme plus spécifique à cette production héraultaise, on retiendra, comme pour la phase précédente, l'existence de vases à col et d'écuelles de grand volume et l'absence de décor. On soulignera également la présence de deux vases à profil continu dotés de préhensions simples disposées au quadrant, et non diamétralement opposées, comme c'est le plus souvent le cas. Sur un exemplaire, on observe une alternance de tétons et de languette. Cette organisation se retrouve, au Barreau de la DevèzeSud, uniquement dans des fosses que nous attribuons, sur d'autres critères, au Chasséen récent. Aussi, lorsque l'on connait le succès que 

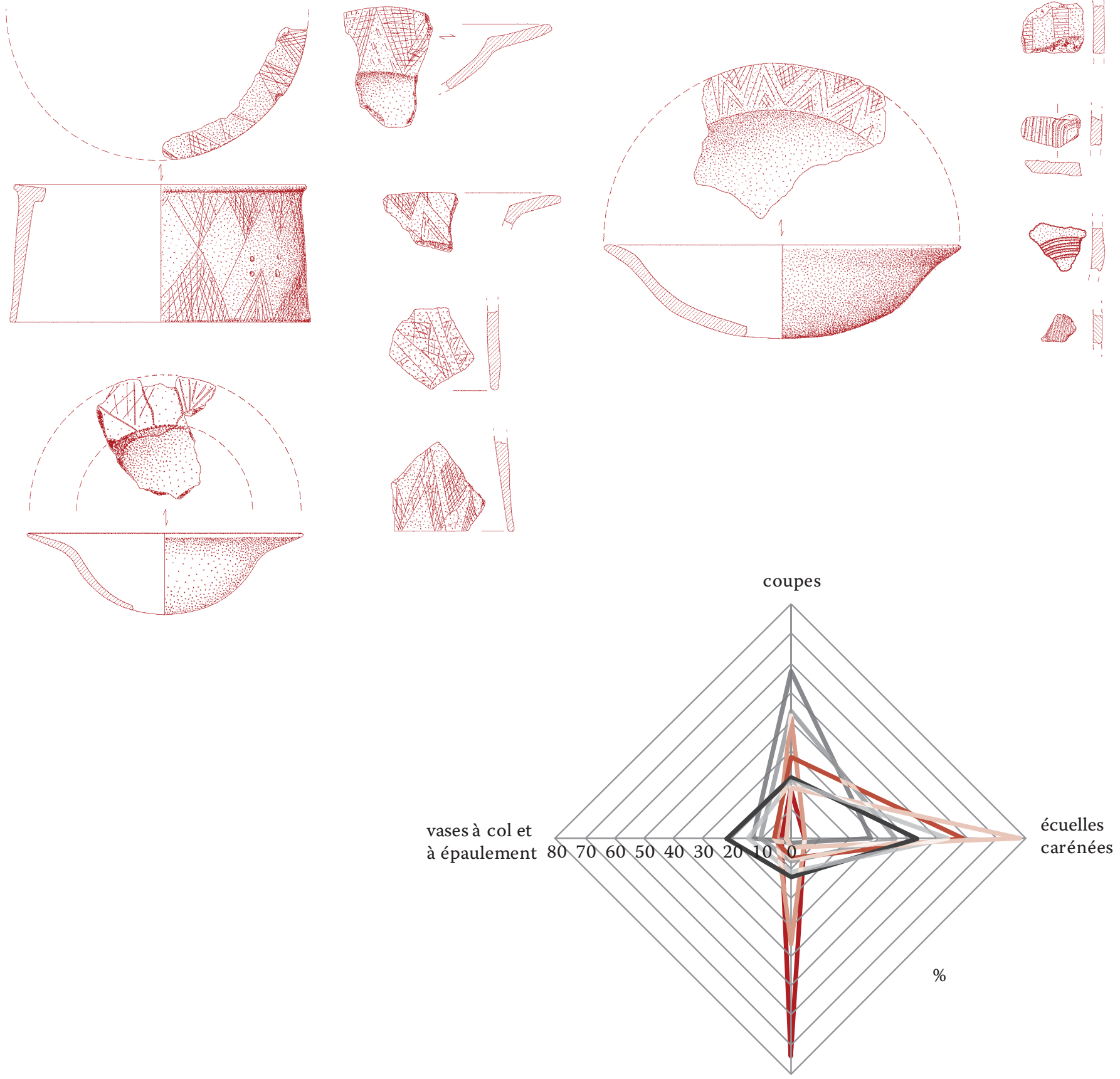

vases globuleux

et tulipiformes

[Fig.5] Principaux décors du Chasséen ancien du Pirou (Valros).

[Fig.6] Graphique en étoile rendant compte de l'évolution de la proportion des principales familles de récipients au sein de huit ensembles du Chasséen méridional.
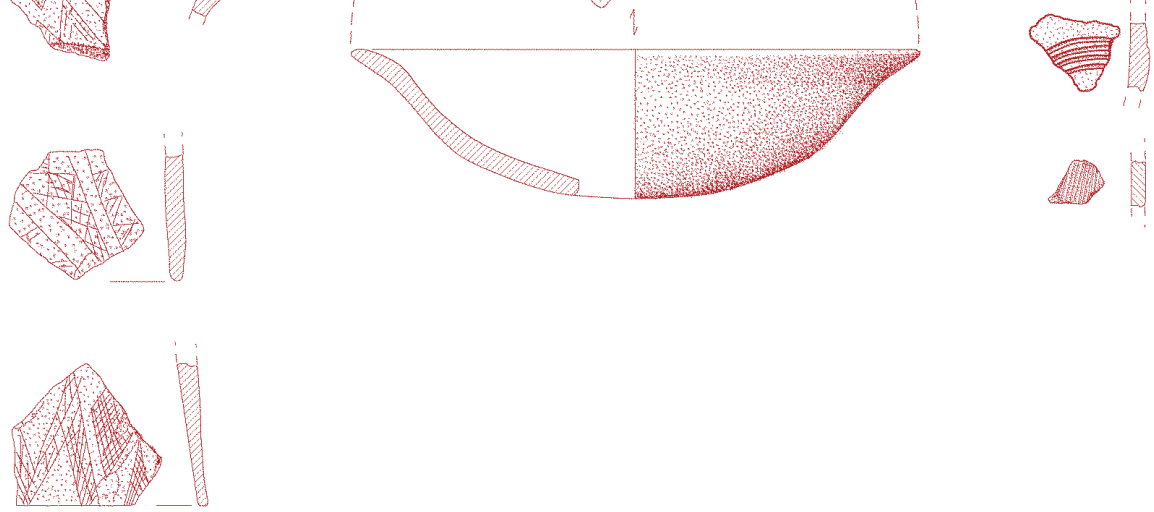

cuelles

vases à col et

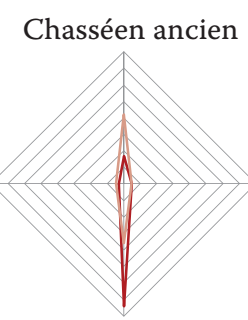

sites

e Crès à Béziers (34) Chasséen ancien

- Fosses du Pirou à Valros (34) Chasséen ancien
Chasséen classique

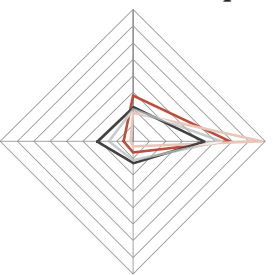

\footnotetext{
Auriac, Carcassonne (11) - Fossé 3B à Cugnaux (31)
- Chasséen "classique"

- Fossé 168b à SaintMichel-du-Touch (31) Chasséen classique Devèze sud à Béziers (34)
- Chasséen classique
}

Chasséen récent

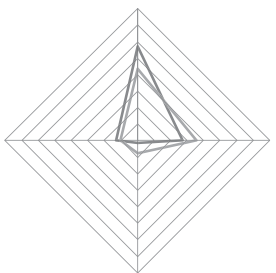

- Fossé 4 à Chateau-Percin (Seilh, 31)

Chasséen récent

- Fossé P17-1 à VilleneuveTolosane (31) -Chasséen récen 
connaîtrons, au Néolithique final (Vérazien), les alignements et les alternances de préhensions simples, on peut s'interroger sur une éventuelle paternité du type qui serait ici mise en évidence.

Les fouilles réalisées ces dix dernières années ont donc considérablement fait évoluer les connaissances relatives aux productions céramiques chasséennes de l'Ouest de l'Hérault. En chronologie relative, ces productions constituent, à l'heure actuelle, le meilleur élément de datation des occupations du Néolithique moyen 2 découvertes dans cette zone. Replacées dans le contexte plus large du Chasséen méridional, ces séries viennent compléter ou affiner les séquences définies par ailleurs et s'insèrent parfaitement dans le schéma global du Chasséen méridional, bien qu'elles ne soient pas exemptes de spécificités locales.

GEORJON C., 2003 : «Chronologie, variabilité et phénomènes de récurrence dans les corpus céramiques chasséens de la basse vallée de Lez (Hérault) ", in GASCO J., Gutherz X., LABRIFfe DE P.-A. (DIR.), Temps et espaces culturels du 6ème au $2^{\text {ème }}$ millénaire en France du Sud, Actes des $4^{\text {emes }}$ Rencontres Méridionales de Préhistoire Récente, Nîmes, 28-29 octobre 200o, Lattes, ADALR, p. 115-134.

JÉDIKIAN G., 2004: « Études préliminaires du mobilier céramique », in Loison et al., p. 311-336.

LoIson G., FABRE V., VILLEMEUR I. (DIR.), 2004 : Habitats préhistoriques en bordure de l'Orb, Le Crès, Rocade nord de Béziers. DFS de fouille, Inrap, 3 vol., $430 \mathrm{p}$.

Loison G., Gandelin M., Haurillon R., Gleize Y., Marsac R., VergéLy H., 2011 : «Dynamiques d'occupations des sols à la Préhistoire récente dans la basse vallée de l'Hérault. Les apport de l'A75, tronçon Pézenas-Béziers », in SÉnÉPART I., PERrIN T., Thirault E., Bonnardin S. (DIR.), Marges, Frontières et transgressions, Actualité de la recherche, Actes des 8e Rencontres Méridionales de Préhistoire Récente à Marseille, 7-8 novembre 2008 , Archives d'Écologie Préhistorique, p. 317-343.

VAQUER J., 1991 : « Aspect du Chasséen en Languedoc occidental : habitat et culture matérielle », in Identité du Chasséen, Actes du colloque international de Nemours, 17-19 mai 1989, p. 27-37.
1 L'étude du macrooutillage du site a été effectuée par Caroline Hamon

2 Erl-15185 $=5525 \pm 42 \mathrm{BP}$

soit 4455-4327 avant notre ère.
Un lot de céramique singulier dans une sépulture du Chasséen ancien

Hélène Vergély, Muriel Gandelin

Inrap, UMR 5608 "Travaux et Recherches Archéologiques sur les Cultures, les Espaces et les Sociétés» Nicolas Garnier

Laboratoire d'analyses physico-chimiques

a sépulture 3036, découverte sur le site du Barreau de la Devèze-Sud, contient une

inhumation primaire réalisée dans une fosse de plan oblong, à vocation strictement funéraire, de 1,60 m de long pour 1,05 m de large, creusée dans un substrat miocène fortement induré [Fig.1]. Ce type d'aménagement n'est pas ordinaire, pour le Chasséen méridional, au sein duquel les sépultures installées dans des structures en réemploi sont beaucoup plus fréquentes.

Le mobilier associé au défunt est, à plus d'un titre, remarquable. Il s'agit d'un dépôt composé de pièces complètes : un grand polissoir et un lot de trois céramiques. Le polissoir à plage est de forme quadrangulaire en grès fin micacé, sa surface active présente une concavité particulièrement régulière et totalement lissée $\mathbf{1}$. Il se trouvait dans la partie sommitale du remplissage, de manière oblique, position témoignant probablement d'un dispositif architectural en matière putrescible. Le mobilier céramique, quant à lui, a été découvert dans le même horizon stratigraphique que l'inhumation. Du point de vue typologique, il s'agit de formes rares : un micro-vase ovoïde à lèvre ourlée extérieurement [Fig.2], disposé au pied du défunt, au contact direct d'une coupe à socle ajourée de quatre fenêtres ; face au défunt, se trouvait également une écuelle carénée peu profonde à carène anguleuse basse, ornée de deux plaquettes en sablier diamétralement opposées [Fig.3].

La datation, réalisée sur les restes osseux, est comprise entre 4.455 et 4327 avant notre ère ${ }^{2}$, ce qui permet une attribution de cet ensemble à la phase ancienne du Chasséen. Dans ce contexte chronologique, la présence d'une écuelle carénée constitue déjà un fait notable puisque cette forme est rare avant 4100 avant notre ère. De plus, le décor qu'elle supporte est sans équivalent régional connu. Les deux autres vases sont également d'un type peu fréquent: de façon générale les coupes à socle et les micro-vases chasséens complets sont peu nombreux et presque exclusivement issus de contextes sépulcraux ou cultuels. Toutefois, si la typologie des pièces découvertes dans la sépulture 3036 est remarquable, l'examen attentif de leur mise en forme tend à montrer que, du point de vue technique, elles n'ont pas fait l'objet d'un surinvestissement de la part des potiers. Même si la taphonomie ne permet pas de postuler sur l'aspect éventuellement poli des surfaces, le modelage apparait assez sommaire, voire grossier surtout pour la coupe à socle et, dans une moindre mesure, l'écuelle. Et l'on est en droit de s'interroger sur l'utilisation d'un mobilier à vocation exclusivement funéraire, probablement à usage unique.

L'association du micro-vase et de la coupe à socle étant exceptionnelle, elle invitait à l'élaboration de multiples hypothèses. Nous avons choisi de 